CASE REPORT

\author{
O.A. Glenn \\ E.M. Quiroz \\ J.I. Berman \\ C. Studholme \\ D. $\mathrm{Xu}$
}

\section{Diffusion-Weighted Imaging in Fetuses with Unilateral Cortical Malformations and Callosal Agenesis}

SUMMARY: DWI was performed in fetuses with callosal agenesis and unilateral cortical malformations. ADC values were retrospectively measured in the developing white matter underlying the cortical malformation and compared with the corresponding contralateral white matter. In all 3 patients, ADC values were lower under the areas of cortical malformation compared with the normal contralateral side. Our findings suggest that there are structural differences in the developing white matter underlying areas of cortical malformation.

ABBREVIATIONS: $A D C=$ apparent diffusion coefficient; $D W I=$ diffusion-weighted imaging
D WI has been used to study normal fetal brain development in utero and offers promise for studying abnormalities of fetal brain development. We recently reported our experience with MR imaging in fetuses with callosal agenesis and observed abnormal cortical infoldings in approximately $40 \%$ of fetuses. ${ }^{1}$ Interestingly, we observed abnormal low T2 signal intensity in the parenchyma underlying the abnormal cortical infoldings in most patients. We, therefore, chose to retrospectively study the parenchyma underlying areas of cortical malformations in these fetuses by using DWI to determine whether there were structural differences in the developing white matter. To use the contralateral side as an internal control for comparison, we chose to examine only those fetuses that had unilateral cortical malformations. Although these cortical malformations were presumed on the initial MR imaging, they were confirmed by either follow-up fetal or postnatal MR imaging in 2 patients. The conventional MR imaging findings of these fetuses have been reported previously. ${ }^{1}$ This study was approved by our institutional review board.

\section{Case Reports}

\section{Patient 1}

A 32-year-old woman was referred for a fetal MR imaging at 21 weeks, 3 days of gestation for sonographically detected agenesis of the corpus callosum, abnormal sulcation in the left frontal lobe, and nodular heterotopia. Fetal MR imaging demonstrated callosal agenesis and abnormal infoldings along the medial left frontal and parietal lobes, consistent with cortical malformation and left atrial periventricular

Received June 17, 2009; accepted after revision July 9.

From the Department of Radiology and Biomedical Imaging, University of California, San Francisco, San Francisco, California

This study was supported by the National Institutes of Health (NIH) Grant No. K23 NS52506 and NIH/NCRR (National Center of Research Resources) UCSF-CTSI Grant No. UL1 RR024131. Its contents are solely the responsibility of the authors and do not necessarily represent the official views of the $\mathrm{NIH}$.

Please address correspondence to Orit A. Glenn, MD, Department of Radiology and Biomedical Imaging, University of California, San Francisco, Neuroradiology Section, 505 Parnassus Ave, Box 0628, San Francisco, CA 94143-0628; e-mail: Orit.Glenn@ radiology.ucsf.edu

Indicates open access to non-subscribers at www.ajnr.org

DOI 10.3174/ajnr.A1863 nodular heterotopia (Fig 1). There was subtle T2 hypointensity in the parenchyma underlying the cortical malformation with absence of the multilayered pattern. Single-shot spin-echo echo-planar DWI was performed on a $1.5 \mathrm{~T}$ scanner during a single maternal breath-hold by using b-values of 0 and $600 \mathrm{~s} / \mathrm{mm}^{2}$ applied in 3 orthogonal directions (TR, 4500 ms; TE, minimum; FOV, $32 \mathrm{~cm}$; matrix, $128 \times 128$; section thickness, $5 \mathrm{~mm}$; skip, $2 \mathrm{~mm}$ ). Images were transferred off-line, and ADC maps were calculated. Regions of interest were placed in the developing white matter underlying the cortical malformation and in contralateral developing white matter on the same section. ADC was $10 \%$ lower in the developing white matter underlying the cortical malformation compared with the contralateral side.

The patient underwent a second fetal MR imaging 7 weeks later, which demonstrated an interval increase in the number and extent of abnormal cortical infoldings in the left frontal and parietal lobes, with persistent subtle T2 hypointensity in the parenchyma underlying the cortical malformations. The difference in ADC between the 2 sides was $16 \%$, with the ADC remaining lower underlying the cortical malformation. The patient elected to terminate the pregnancy, and postmortem examination was not performed.

\section{Patient 2}

A 36-year-old woman presented with bilateral ventriculomegaly and suspected agenesis of the corpus callosum on sonography performed at 26 weeks, 1 day of gestation. Fetal MR imaging was performed at 28 weeks, 6 days of gestation and demonstrated callosal agenesis, ventriculomegaly, and multiple abnormal infoldings of the right frontal and parietal lobes and insula, consistent with cortical malformation (Fig 2). Subtle T2 hypointensity in the developing white matter underlying the abnormal infoldings was identified. DWI was performed as in case 1, and ADC maps were calculated. Regions of interest were drawn in the developing white matter underlying the right cortical malformation and in corresponding white matter in the left brain on the same section. ADC values were $20 \%$ lower in the developing white matter underlying the cortical malformation compared with the contralateral side. The patient elected to terminate the pregnancy; postmortem examination was declined.

\section{Patient 3}

A 22-year-old woman underwent sonography at 26 weeks, 1 day of gestation, which demonstrated multiple abnormalities, including callosal agenesis, a large interhemispheric cyst, absent vermis, and mild 

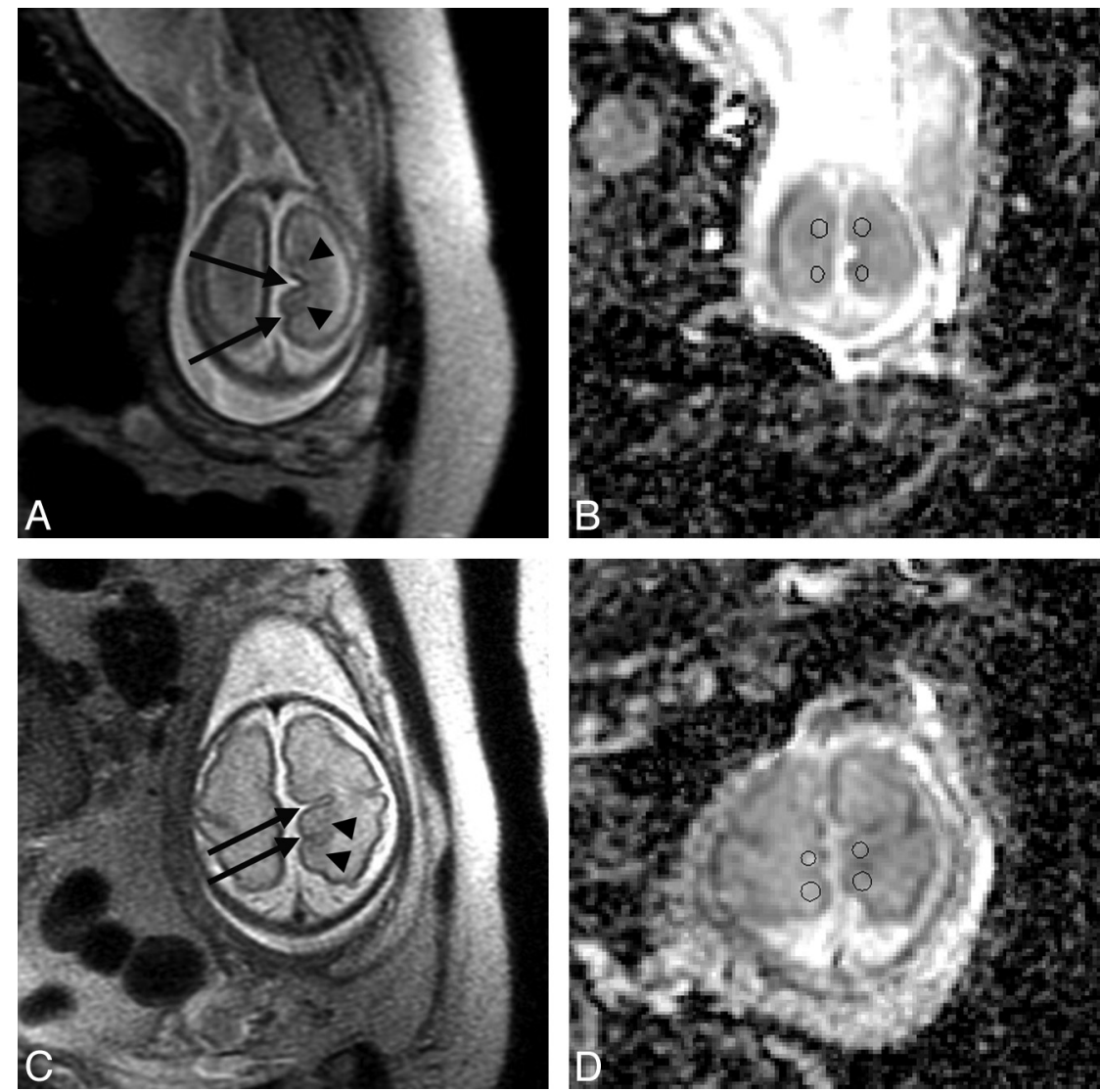

Fig 1. Patient 1. A, Abnormal infoldings along the left frontal and parietal lobes (arrows), with subtle $\mathrm{T} 2$ hypointensity in the underlying developing white matter (arrowheads), are seen on the axial single-shot fast spin-echo T2-weighted image. $B$, Regions of interest are drawn on the corresponding ADC map in the white matter underlying the cortical malformation and in the corresponding contralateral white matter. C, Follow-up scan demonstrates increased extent of the cortical malformation in the left frontal and parietal lobes (arrows), with persistent T2 hypointensity in the underlying white matter (arrowheads). D. Corresponding ADC map with regions of interest in the white matter underlying the cortical malformation and in the corresponding contralateral white matter.
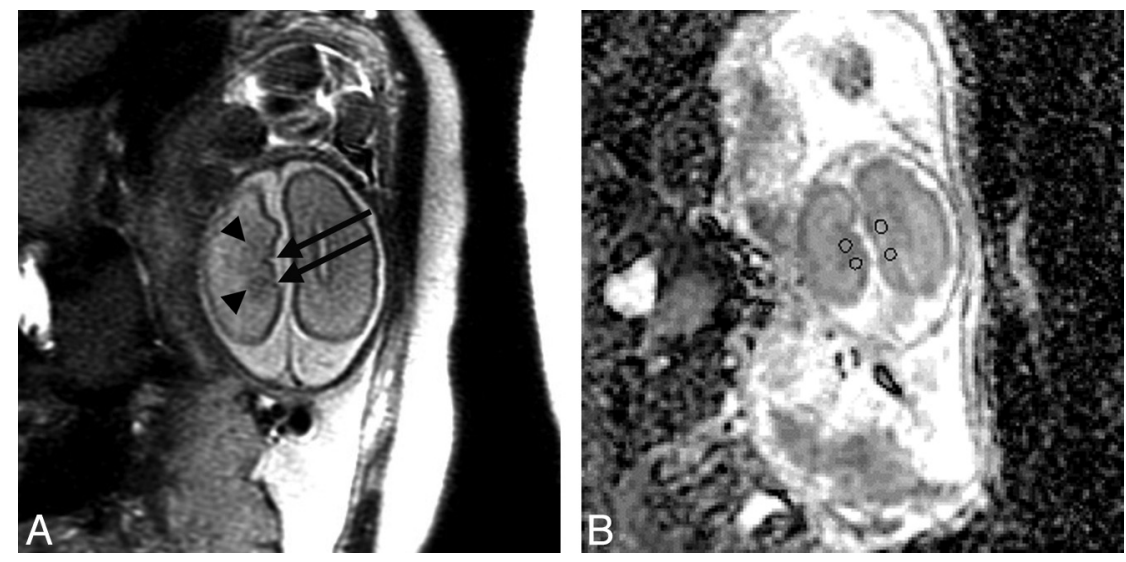

Fig 2. Patient 2. A, Axial single-shot fast spin-echo T2-weighted image demonstrates several abnormal infoldings along the medial right frontal and parietal lobes (arrows), with subtle T2 hypointensity in the underlying developing white matter (arrowheads). B, Regions of interest are drawn on the corresponding ADC map in the white matter underlying the area of abnormal cortical infoldings and in the corresponding contralateral white matter.

ventriculomegaly. Fetal MR imaging performed on the same day confirmed these findings and identified multiple abnormal infoldings of the right frontal, parietal, and temporal lobes, consistent with extensive cortical malformation (Fig 3). There was subtle T2 hypointensity in the right frontal, parietal, and temporal lobes, with a lack of a normal multilayered pattern. Additional abnormalities identified by fetal MR imaging included right atrial periventricular nodular heterotopia, a small and dysmorphic brain stem and cerebellar hemi- spheres, and cleft palate. DWI was performed as in case 1, and regions of interest were placed in the parenchyma underlying the areas of cortical malformation and in contralateral areas on the same section. ADC values were $8.7 \%$ lower in the developing white matter underlying the cortical malformation compared with the contralateral white matter. The neonate was delivered at term and diagnosed with orofacial digital syndrome type I. Postnatal MR imaging performed at 3 months of age confirmed the fetal MR imaging findings. 

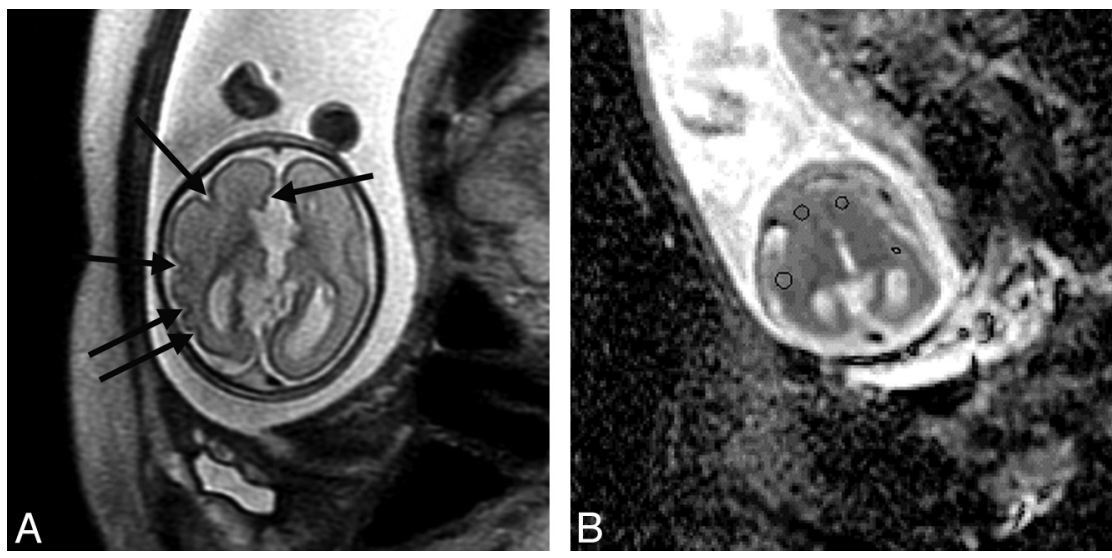

Fig 3. Patient 3. A, Axial single-shot fast spin-echo T2-weighted image demonstrates extensive abnormal infoldings in the right temporal, parietal, and frontal lobes (arrows). There is subtle T2 hypointensity in the underlying white matter. $B$, Regions of interest are drawn on the corresponding ADC map in the white matter underlying the areas of abnormal cortical infolding and in the corresponding contralateral white matter.

\section{Discussion}

We observed lower ADC values in the white matter underlying areas of cortical malformation compared with the contralateral normal-appearing side in fetuses with unilateral cortical malformations and callosal agenesis. Previous studies on normative fetal ADC values have demonstrated no significant difference between right and left ADC values. ${ }^{2,3}$ Thus, our findings suggest that the development of the white matter subjacent to cortical malformations is different from that on the contralateral side, perhaps in part due to abnormal organization and increased cellularity in the developing white matter. Indeed, heterotopic neurons have been observed in the white matter subjacent to cortical malformations in postmortem studies of children with polymicrogyria. ${ }^{4,5}$ In addition, neuronal heterotopia has also been observed in the white matter adjacent to areas of cortical malformation in an autopsy study of a 21-week-gestation fetus with callosal agenesis and orofacial digital syndrome type VI. ${ }^{6}$

Our findings are interesting because previous diffusion studies of cortical malformations have focused on patients with epilepsy and cortical malformations. These studies have primarily used diffusion tensor imaging and have shown that fractional anisotropy values are decreased and ADC values are unchanged or increased in areas of cortical malformations and adjacent white matter compared with contralateral normalappearing brain and/or control values. ${ }^{7-11}$ Although we were only able to perform DWI and measure ADC values, our findings differ from what has been observed in children and suggest that there are structural differences in the white matter underlying cortical malformations in fetuses compared with children. Most interesting, low ADC values were also observed in the parenchyma of a 32-gestational-week fetus with hemimegalencephaly. ${ }^{12}$

It is possible that the effects of chronic seizures occurring in childhood result in the differences observed between fetuses and children. More specifically, animal models have shown that status epilepticus results in cell degeneration and death, even in chronic stages. ${ }^{13}$ Moreover, studies have shown increased ADC values in the chronic phase in animals with status epilepticus. ${ }^{14,15}$ Thus, the chronic effects of repeated childhood seizures could result in increased ADC values, even if there is increased cellularity underlying the cortical malformations in the fetus.

In summary, we observed lower ADC values in the developing fetal white matter underlying areas of cortical malformation compared with the contralateral developing white matter. Future studies using diffusion tensor imaging in the fetus and neonate are needed to better understand these structural differences as well as changes that may occur during gestation and childhood.

\section{References}

1. Tang PH, Bartha AI, Norton ME, et al. Agenesis of the corpus callosum: an MR imaging analysis of associated abnormalities in the fetus. AJNR Am J Neuroradiol 2009;30:257-63

2. Schneider JF, Confort-Gouny S, Le Fur Y, et al. Diffusion-weighted imaging in normal fetal brain maturation. Eur Radiol 2007;17:2422-29

3. Schneider MM, Berman JI, Baumer FM, et al. Normative apparent diffusion coefficient values in the developing fetal brain. AJNR Am J Neuroradiol 2009; 30:1799-803. Epub 2009 Jun 25

4. Billette de Villemeur T, Chiron C, Robain O. Unlayered polymicrogyria and agenesis of the corpus callosum: a relevant association? Acta Neuropathol 1992;83:265-70

5. Van den Veyver IB, Panichkul PP, Antalffy BA, et al. Presence of filamin in the astrocytic inclusions of Aicardi syndrome. Pediatr Neurol 2004;30:7-15

6. Lesca G, Fallet-Bianco C, Plauchu H, et al. Orofaciodigital syndrome with cerebral dysgenesis. Am J Med Genet A 2006;140:757-63

7. Bonilha L, Halford J, Rorden C, et al. Microstructural white matter abnormalities in nodular heterotopia with overlying polymicrogyria. Seizure 2007;16: 74-80. Epub 2006 Nov 13

8. Eriksson SH, Rugg-Gunn FJ, Symms MR, et al. Diffusion tensor imaging in patients with epilepsy and malformations of cortical development. Brain 2001;124:617-26

9. Trivedi R, Gupta RK, Hasan KM, et al. Diffusion tensor imaging in polymicrogyria: a report of three cases. Neuroradiology 2006;48:422-27

10. Widjaja E, Blaser S, Miller E, et al. Evaluation of subcortical white matter and deep white matter tracts in malformations of cortical development. Epilepsia 2007;48:1460-69

11. Wieshmann UC, Clark CA, Symms MR, et al. Reduced anisotropy of water diffusion in structural cerebral abnormalities demonstrated with diffusion tensor imaging. Magn Reson Imaging 1999;17:1269-74

12. Agid R, Lieberman S, Nadjari M. Prenatal MR diffusion-weighted imaging in a fetus with hemimegalencephaly. Pediatr Radiol 2006;36:138-40

13. Wasterlain CG, Niquet J, Thompson KW, et al. Seizure-induced neuronal death in the immature brain. Prog Brain Res 2002;135:335-53

14. Grohn OHJ, Pitkanen A. Magnetic resonance imaging in animal models of epilepsy: noninvasive detection of structural alterations. Epilepsia 2007;48:3-10

15. Nairismagi J, Grohn OHJ, Kettunen MI, et al. Progression of brain damage after status epilepticus and its association with epileptogenesis: a quantitative MRI study in a rat model of temporal lobe epilepsy. Epilepsia 2004;45:1024-34 